\title{
Neurons within the Same Network Independently Achieve Conserved Output by Differentially Balancing Variable Conductance Magnitudes
}

\author{
Joseph L. Ransdell, ${ }^{1}$ Satish S. Nair, ${ }^{2}$ and David J. Schulz ${ }^{1}$ \\ Departments of ${ }^{1}$ Biological Sciences and ${ }^{2}$ Electrical and Computer Engineering, University of Missouri, Columbia, Missouri 65211
}

\begin{abstract}
Biological and theoretical evidence suggest that individual neurons may achieve similar outputs by differentially balancing variable underlying ionic conductances. Despite the substantial amount of data consistent with this idea, a direct biological demonstration that cells with conserved output, particularly within the same network, achieve these outputs via different solutions has been difficult to achieve. Here we demonstrate definitively that neurons from native neural networks with highly similar output achieve this conserved output by differentially tuning underlying conductance magnitudes. Multiple motor neurons of the crab (Cancer borealis) cardiac ganglion have highly conserved output within a preparation, despite showing a 2-4-fold range of conductance magnitudes. By blocking subsets of these currents, we demonstrate that the remaining conductances become unbalanced, causing disparate output as a result. Therefore, as strategies to understand neuronal excitability become increasingly sophisticated, it is important that such variability in excitability of neurons, even among those within the same individual, is taken into account.
\end{abstract}

\section{Introduction}

Neurons with similar and conserved output across individuals can exhibit 2-6-fold variability in their intrinsic conductances (Golowasch et al., 2002; Swensen and Bean, 2005; Schulz et al., 2006; Goaillard et al., 2009; Ransdell et al., 2012) as well as in ion channel mRNA levels (Schulz et al., 2006, 2007). This observation has motivated several computational studies that used populations of model cells with variable intrinsic properties and constrained output features to demonstrate that model neurons could achieve similar voltage output with disparate intrinsic parameters (Goldman et al., 2001; Prinz et al., 2004; Taylor et al., 2009; Ball et al., 2010). This approach has complemented and extended accumulating biological evidence that is consistent with the idea that output features across identified cells may be conserved by both balance and compensation among key intrinsic conductances, rather than by tightly regulating the magnitude of individual conductances (MacLean et al., 2003; Swensen and Bean, 2005; Grashow et al., 2010; Ransdell et al., 2012). However, most of these studies involve compensation that leads to conservation of output following perturbation of the cellular excitability (MacLean et al., 2003; Bergquist et al., 2010), often via gene knock-out (Van Wart and Matthews, 2006; Nerbonne

\footnotetext{
Received March 12, 2013; revised April 22, 2013; accepted May 8, 2013.

Author contributions: J.L.R., S.S.N., and D.J.S. designed research; J.L.R. performed research; J.L.R. and D.J.S. analyzed data; J.L.R., S.S.N., and D.J.S. wrote the paper.

This work was supported by the Missouri Spinal Cord Injuries Program, and DoD CDMRP hypothesis and exploration award No. SC090555.

The authors declare no competing financial interests.

Correspondence should be addressed to Dr David J. Schulz, 218a LeFevre Hall, University of Missouri-Columbia, Columbia, M0 65211. E-mail:SchulzD@missouri.edu.

DOI:10.1523/JNEUROSCI.1095-13.2013

Copyright $\odot 2013$ the authors $\quad 0270-6474 / 13 / 339950-07 \$ 15.00 / 0$
}

et al., 2008) or pharmacological manipulation (Swensen and Bean, 2005; Ransdell et al., 2012). Although these studies demonstrate that variability could arise as a result of such homeostatic plasticity, these studies do not address intrinsic variability found in these neurons with all conductances intact, or whether this variability represents differential tuning to conserved output in vivo.

We use the crustacean cardiac ganglion, which contains multiple large cell (LC) motor neurons that produce synchronous bursting output, to test whether individual neurons achieve conserved output by differentially balancing conductance magnitudes. We examine multiple motor neurons within the same ganglia, which are genetically identical and have similar output in the native network, and find that they have varying underlying conductances. Then by disrupting a select subset of conductances in these cells with pharmacological blockade, we demonstrate a corresponding disruption in conserved output. We believe this is the first direct demonstration that multiple neurons, particularly within the same neural network, naturally generate similar output by differentially balancing variable underlying conductances, independent of induced compensatory changes.

\section{Materials and Methods}

Animals. Crabs (Cancer borealis) of either sex were purchased and shipped overnight from The Fresh Lobster Company. Crabs were kept at $12^{\circ} \mathrm{C}$ in artificial seawater. Dissections took place in chilled physiological saline: $440 \mathrm{~mm} \mathrm{NaCl}, 26 \mathrm{~mm} \mathrm{MgCl} 213 \mathrm{~mm} \mathrm{CaCl} 2,11 \mathrm{~mm} \mathrm{KCl}$, and $10 \mathrm{~mm}$ HEPES, pH 7.4. Chemicals were obtained from Fisher Scientific unless otherwise noted.

Electrophysiology. All intracellular recordings were made from LC3, 4, and 5 somata. LC structure consists of a cell soma and proximal neurite 

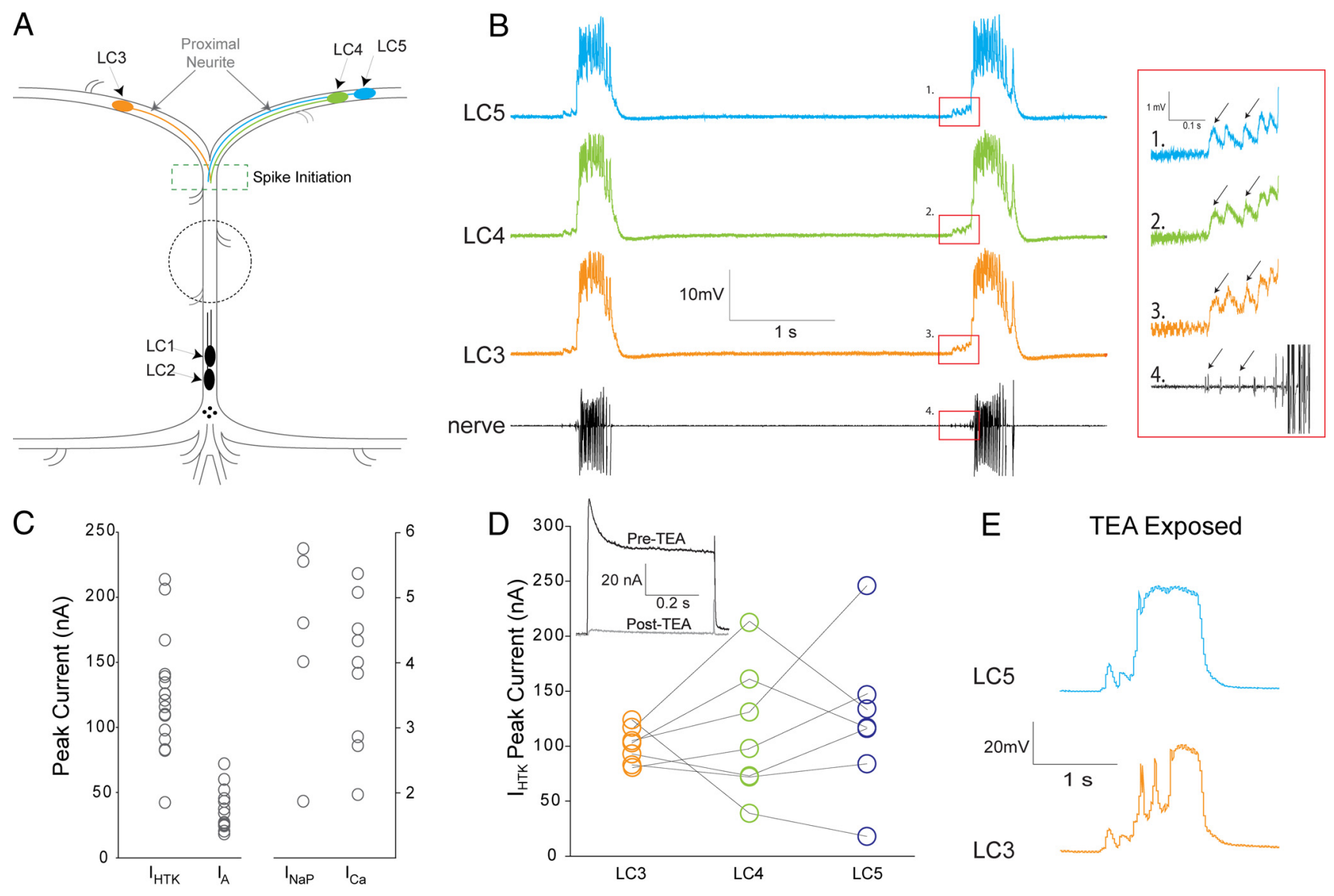

$\mathrm{F}$

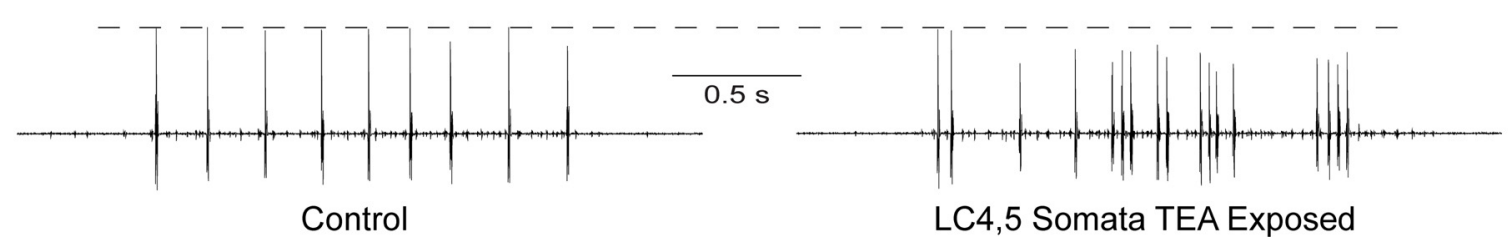

Figure 1. A, Schematic of C. borealis cardiac ganglion showing location of $\mathrm{L}($ somata (ovals) and pacemaker interneurons (small circles). Extracellular recordings are taken from the central nerve (dashed circle). All experiments were conducted on three anterior LCs 3-5 (colored ovals). These cells project a proximal neurite from their somata toward a common spike initiation zone region (green dashed box), and then axons continue posterior. B, Activity of $\mathrm{LC}$ neurons during spontaneous network activity. Extracellular recording (bottom trace) shows $\mathrm{LC}$ (tall spike) and pacemaker (short spike) APs. Inset, Red box shows enlarged recordings displaying that each pacemaker AP (small spike) results in an EPSP in all three anterior LCs. Two pacemaker APs and resulting EPSPs are

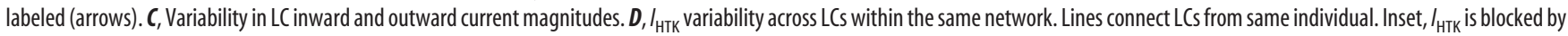
$25 \mathrm{~mm}$ TEA exposure $(30 \mathrm{~min}$ ). Current traces before (black) and after (gray) TEA were clamped at $0 \mathrm{mV}$ step from $-40 \mathrm{mV}$ holding potential. $\boldsymbol{E}$, TEA exposure in the intact network desynchronizes membrane potential waveforms of $L C s$ during ongoing rhythmic activity. $F$, Spiking output (extracellular recordings) is affected by TEA applied to LC4 and LC5 somata. Dashed line for comparison of spike amplitudes. Traces were taken from the same recording well within 10 min of one another.

that projects to a common spike initiation area located posterior to the anterior branch point (Hartline, 1967; Tazaki and Cooke, 1983; Cooke, 2002; Ransdell et al., 2013) (Fig. 1A). Somata were separated from action potential (AP) conductances (unless noted otherwise) by tightening a thread ligature past the anterior branch point on the nerve the LC was located in (Figs. 1A, 2A,B). Isolated somata carry ionic conductances that generate driver potential output (Tazaki, 1972; Tazaki and Cooke, 1983, 1986; Cooke, 2002), even with ligatures as close as $200 \mu \mathrm{m}$ from the soma (Tazaki and Cooke, 1983).

Experiments were performed in physiological saline at $12^{\circ} \mathrm{C}$. Extracellular recordings were made with stainless steel pin electrodes in petroleum jelly wells placed on the nerve. Signals were amplified and filtered using a differential AC amplifier (A-M Systems). Intracellular recordings were made with glass electrodes containing $3 \mathrm{M} \mathrm{KCl}(8-17 \mathrm{M} \Omega)$ using an Axoclamp 2A amplifier (Molecular Devices). Two-electrode voltageclamp (TEVC) and two-electrode current-clamp (TECC) protocols were created, driven, and recorded with Clampex 9.2 software (Molecular
Devices). Recordings were analyzed with Clampfit 9.2 (Molecular Devices). $R_{\text {in }}$ was measured in TECC with 10 negative current injection steps $(t=2 \mathrm{~s},-1$ to $-10 \mathrm{nA})$.

TEVC measurements for $I_{\mathrm{A}}, I_{\mathrm{HTK}}, I_{\mathrm{Ca}}$, and $I_{\mathrm{NaP}}$ were made using protocols described in detail in our previous work (Ransdell et al., 2012, 2013). Outward currents were measured at $0 \mathrm{mV}$, inward currents at peak conductance.

Stimulus protocols. To generate current injection waveforms, we recorded voltage waveforms from LC somata during intact network activity. For Stimulus Protocol 1, this consisted of $20 \mathrm{~s}$ recordings from LC3 that included four burst potentials with both pacemaker EPSPs and LC back-propagating APs (BPAPs). During this recording $V_{\text {rest }}=-55 \mathrm{mV}$. For Stimulus Protocol 2, to remove LC BPAPs, we hyperpolarized the LC soma to $-65 \mathrm{mV}$ to eliminate spiking. This enabled us to record the voltage change caused by a single burst of pacemaker EPSPs. We then isolated LC3 with ligature and used the voltage waveforms recorded as the command voltage in TEVC. The average current injection over five 


\section{A Stimulus protocol 1 (synaptic + BPAPs)}
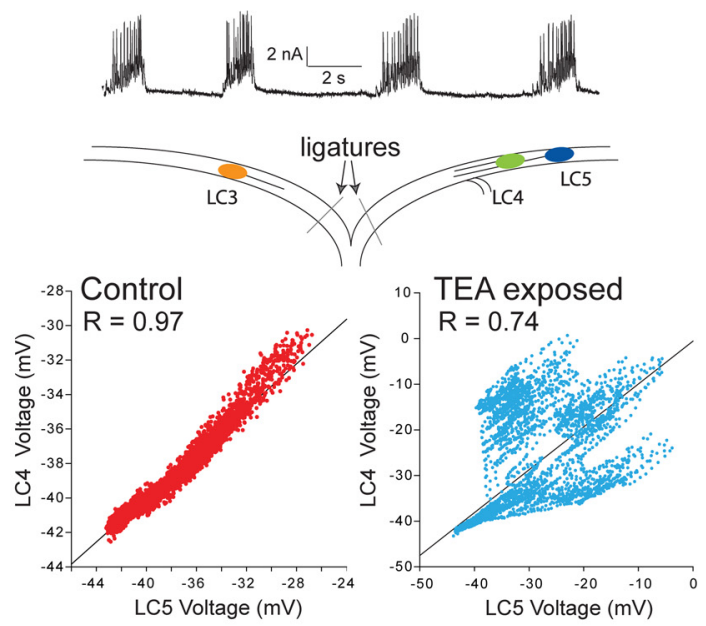
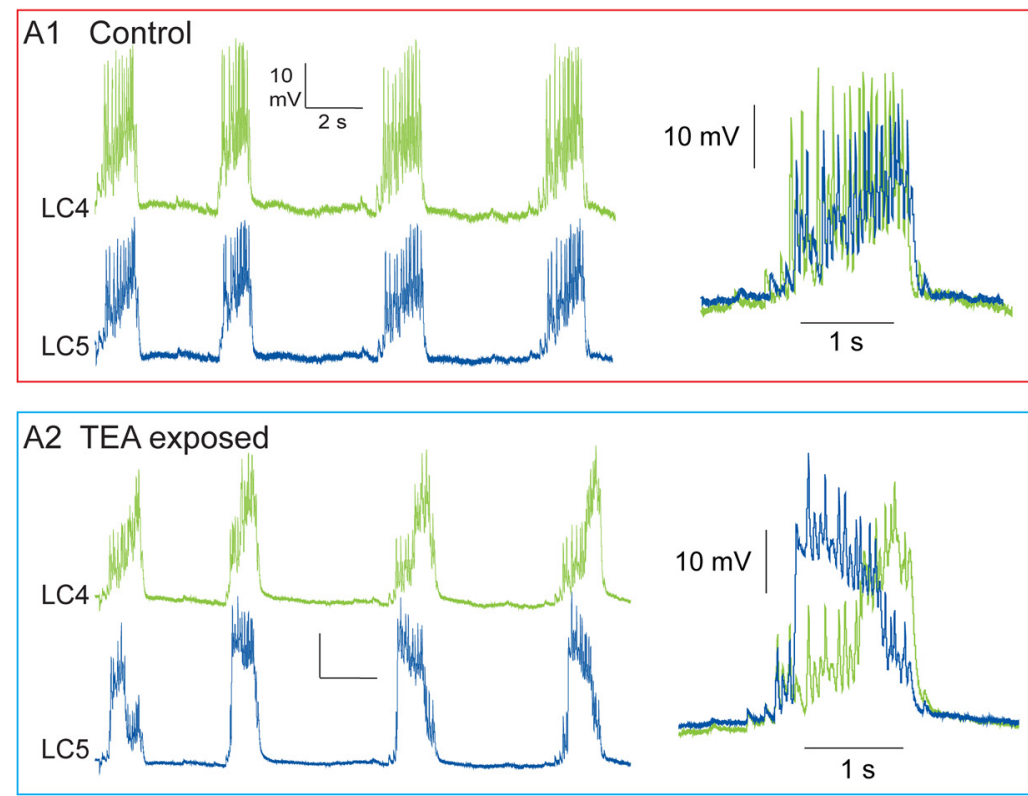

\section{B Stimulus protocol 2} (synaptic only)
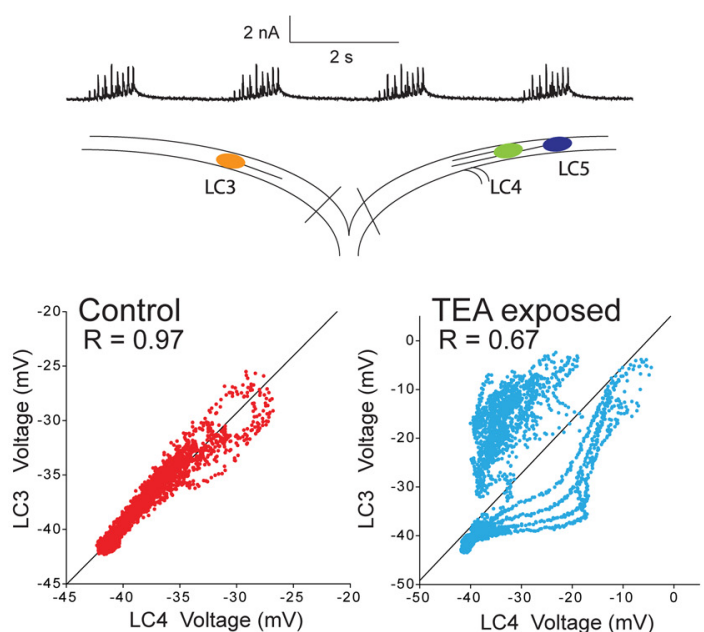
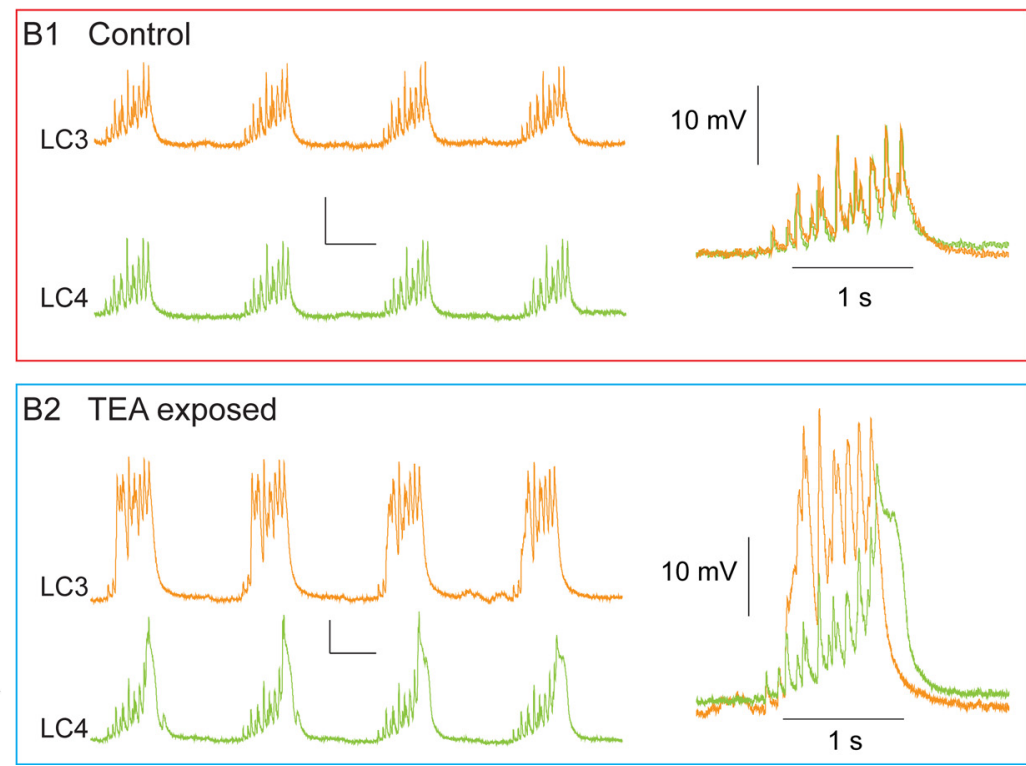

Figure 2. Realistic current injection protocols used to test intrinsic excitability of isolated LCs. $A$, Stimulus protocol 1 reproduces both synaptic currents and back propagating APs (BPAPs). LCs 3 , 4 , and 5 are isolated from the network via thread ligatures (slash marks), and the stimulus protocol is applied before (A1, control, red box) and after (A2-TEA exposed, blue box) exposure to 25 mM TEA. Representative traces show the response to the entire stimulus protocol, and overlaid traces show the fourth burst in the sequence. Scatterplots show pairwise correlation of time matched voltages (sampled at $500 \mathrm{~Hz}$ ) of the waveforms shown in the representative traces. $R$ values are results of Pearson's correlation test for these two cells. Loss of conserved output is demonstrated by the lack of correlation in the scatterplot as well as the decrease in $R$ value. $B$, Stimulus Protocol 2 reproduces synaptic currents $L C s$ receive from pacemaker neurons. Experiments and analyses were performed as in Stimulus Protocol 1.

sweeps necessary to generate each of the voltage waveforms was used for Stimulus Protocols 1 and 2.

Each stimulus protocol was applied at $V_{\text {rest }}$ and four starting voltages $(-60,-50,-40,-30 \mathrm{mV})$ controlled with DC current injection. Blockers (25 mM TEA or $1 \mathrm{~mm} 4 \mathrm{AP}$ ) were applied to the preparation for $30 \mathrm{~min}$, after which stimulus protocols were repeated at each starting voltage.

Statistics. Analyses used SigmaPlot 11.0 and Clampfit 9.2 software. $R$ values are results of Pearson correlation (Fig. 2) or cross-correlation function estimate (Figs. 3, 4) with a $10 \mathrm{~ms}$ lag; in cross-correlation analysis, the peak $R$ value across the lag period was used. Correlation analyses were conducted only between LCs of the same ganglion using the entire voltage trace from each stimulus protocol. After failing Shapiro-Wilk tests for normality, changes in $R_{\mathrm{in}}, V_{\text {rest }}$, and differences between $R$ values before and after pharmacological blockade were analyzed with MannWhitney U tests.

\section{Results}

In the intact cardiac ganglion (Fig. $1 A$ ), bursts in LCs are initiated via excitatory input from pacemaker interneurons (Fig. $1 B$ ) that result in synchronous burst and AP generation in all five LC motor neurons (Fig. 1B). In addition, LC somata contain the intrinsic conductances that influence burst potentials (Fig. 1C). However, the magnitudes of these currents vary not only across animals (Fig. 1C), but even among cells of the same ganglion (Fig. $1 D)$. If variability in current magnitude suggests that individual 
A

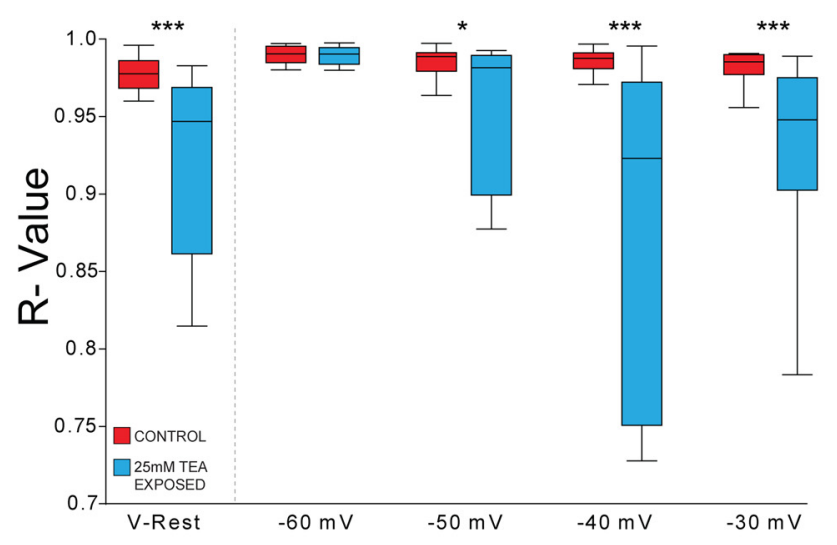

C

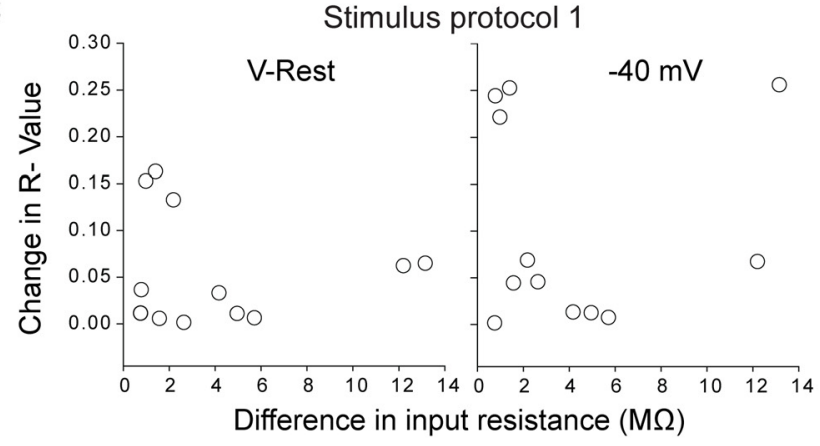

$\mathrm{E}$

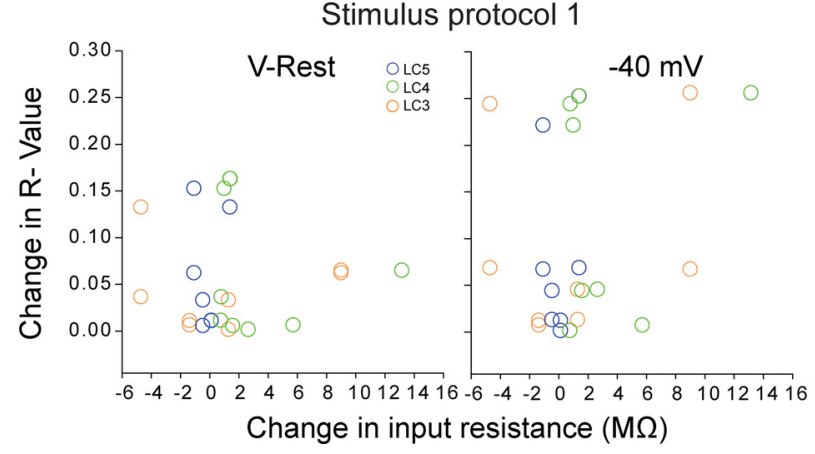

B

Stimulus protocol 2
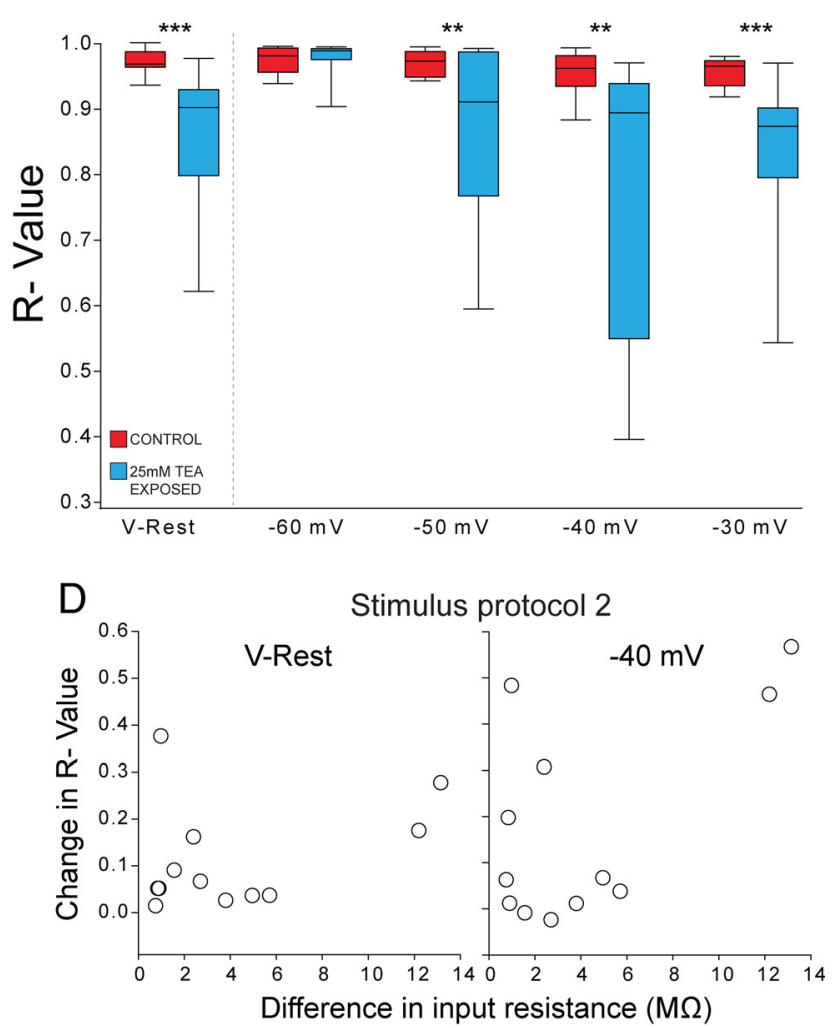

$\mathrm{F}$

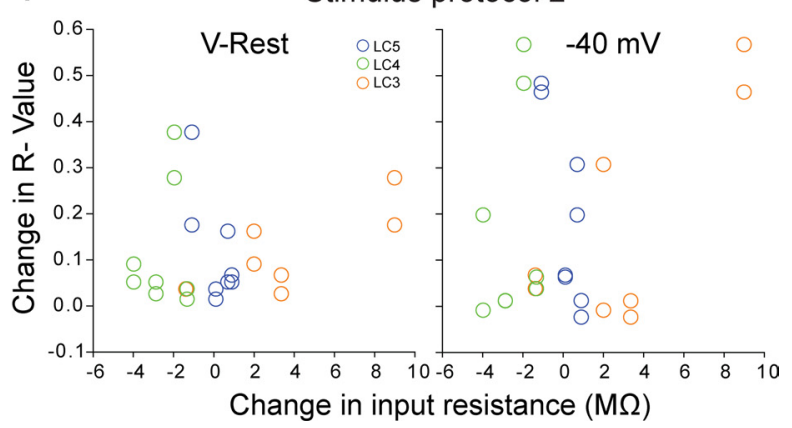

Figure 3. Changes in output after TEA exposure. $A, B, B$ ox plots show distributions of $R$ values from cross-correlation analysis of $L C$ voltage waveforms generated by Stimulus Protocol 1 ( $A$ ) and Stimulus Protocol 2 ( $\boldsymbol{B}$ ) before (red boxes) and after (blue boxes) $25 \mathrm{~mm}$ TEA exposure. Lines within boxes mark the median, box boundaries represent $25^{\text {th }}$ and $75^{\text {th }}$ percentiles, and whiskers represent maximum and minimum values. All comparisons were made between $L C s$ of the same individual ( $N=12$ comparisons across 6 individuals). Each stimulus was injected into an isolated $\mathrm{LC}$ at its natural $V_{\text {rest }}$ and four starting voltages. Significant differences in median $R$ value were tested with Mann-Whitney $\mathrm{U}$ tests, and are denoted by asterisks $\left({ }^{* * *} p<0.005,{ }^{* *} p<0.01,{ }^{*} p<\right.$ $0.05)$. C, D, Comparison of change in $R$ value with difference in $R_{\text {in }}$ between $L($ s after TEA exposure for Stimulus Protocol $1(\boldsymbol{C})$ and Stimulus Protocol 2 (D). E, F, Comparison of change in $R$ value with change in $R_{\text {in }}$ after TEA exposure for Stimulus Protocol $1(\boldsymbol{E})$ and Stimulus Protocol $2(\boldsymbol{F})$.

cells differentially balance their conductances to achieve a common output, then blocking a subset of conductances should reveal differential tuning of the remainder, as demonstrated by a shift away from conserved output. Our initial data were consistent with this hypothesis: when LCs were exposed to highthreshold $\mathrm{K}^{+}$current $\left(I_{\mathrm{HTK}}\right)$ blocker (TEA; Fig. $1 D$, inset), their normally synchronous waveforms in spontaneously active networks became desynchronized and variable (Fig. 1E). TEA application localized to LC somata is able to affect spiking output, causing changes in the spiking pattern as well as apparent desynchronization of spiking as revealed by a decrease in spike amplitude that we interpret as loss of summed, synchronous AP activity (Fig. $1 F$ ). This decrease in amplitude cannot be attributed to TEA block of APs, as the somata localization of the TEA in this experiment left axonal conductances intact. These data demonstrate that LC somatic conductances play a significant role in shaping waveform as well as spiking output in these cells.

Because cells in intact networks are subject to many factors that influence output, we used an experimental strategy to test solely the influence of variable conductances on output in neurons isolated from their constituent network connections. To reliably demonstrate a shift away from conserved output at the single cell level following conductance block, we devised standardized, biologically relevant current injection protocols. We isolated the somata of the three anterior LCs from pacemaker synaptic input, electrical coupling, and BPAPs, effectively isolating their burst generating conductances (Fig. $2 A, B$ ). We then used current injection to characterize cell output before and after 
blocking conductances. In designing the protocol, which employs somatic current injections, we considered that somata naturally receive two kinds of current input: synaptic and BPAPs. We recorded these somatic input currents from cells in intact networks, and subsequently used these recordings as the basis for realistic current injections applied to isolated cell somata (Fig. 2A,B; see Materials and Methods). Stimulus Protocol 1 consists of both biologically realistic synaptic currents plus BPAPs (Fig. 2A). Stimulus Protocol 2 consists solely of current injection of synaptic drive to the soma via pacemaker inputs (Fig. 2B). We first determined that our current injection protocols, when applied to isolated somata with all intrinsic conductances intact, produced virtually identical voltage responses across LCs as measured by cross-correlation analysis. Under control conditions, current injection elicited conserved membrane waveforms across LCs in the same network (Fig. 2 A1, B1, controls). Thus the basis for our experiments is whether these conserved output responses are maintained subsequent to pharmacological blockade of conductances.

We considered four key points in testing the hypothesis of conserved output from variable underlying conductances. First, we ensured that individual conductance levels do indeed vary across isolated cells. We measured current levels carried by four ionic conductances in LC somata: $I_{\text {HTK }}$ (high-threshold $\mathrm{K}^{+}$current, a combination of $I_{\mathrm{Kd}}$ and $I_{\mathrm{KCa}}$ ), $\mathrm{I}_{\mathrm{A}}$ (transient $\left.\mathrm{K}^{+}\right), I_{\mathrm{Ca}}$, and $I_{\mathrm{NaP}}$ (persistent $\mathrm{Na}^{+}$). Each current measured varied at least 2-4-fold across individual cells (Fig. 1C). Second, if output simply were constrained by electrical synapses between LCs, then isolated neurons should no longer produce conserved output from standardized current injection. This was not the case; isolated LCs with all conductances intact produced remarkably consistent output (Fig. 2 , controls). Third, if conserved output is simply a result of isolated cells passively following current injection, then blocking conductances should not alter this output. This also was not the case; when $I_{\mathrm{HTK}}$ is blocked with TEA (Fig. 1D), cell output as a result of the standardized current injection was substantially altered (Fig. 2 A2, B2, blue boxes). Finally, and perhaps most pertinent to the hypothesis, if cells are not differentially tuned to produce similar output, then blocking a conductance might still be expected to alter output, but should produce uniform changes across cells. This was not the case; when TEA was applied to isolated LCs, and standardized current injection applied, the once similar outputs diverge between cells (Fig. 2) as revealed by significantly lower (and highly variable) $R$ values from cross-correlation analyses following TEA exposure (Fig. $3 A, B$ ). To ensure that these differences

B

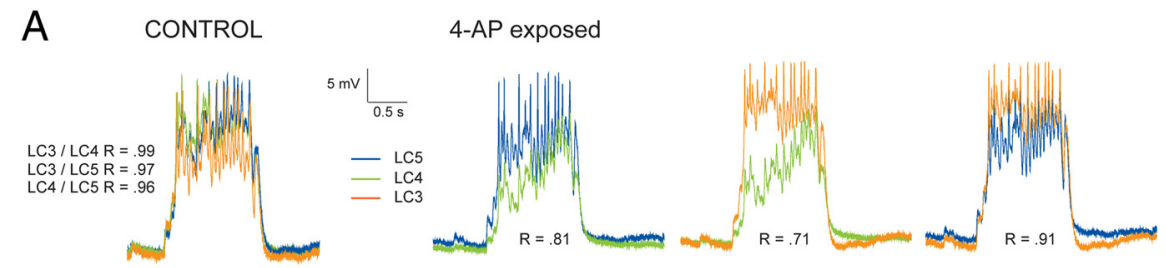

Stimulus protocol 1

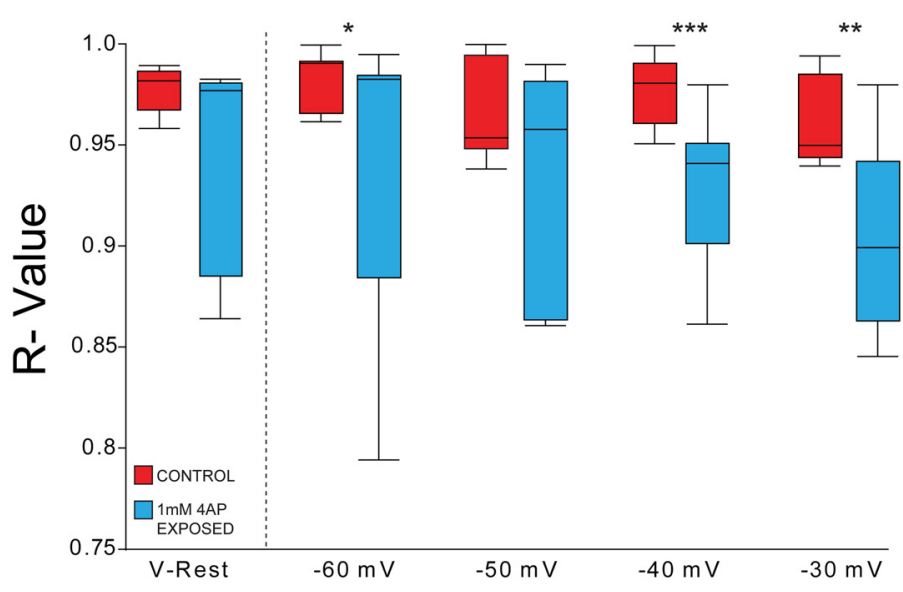

Stimulus protocol 2

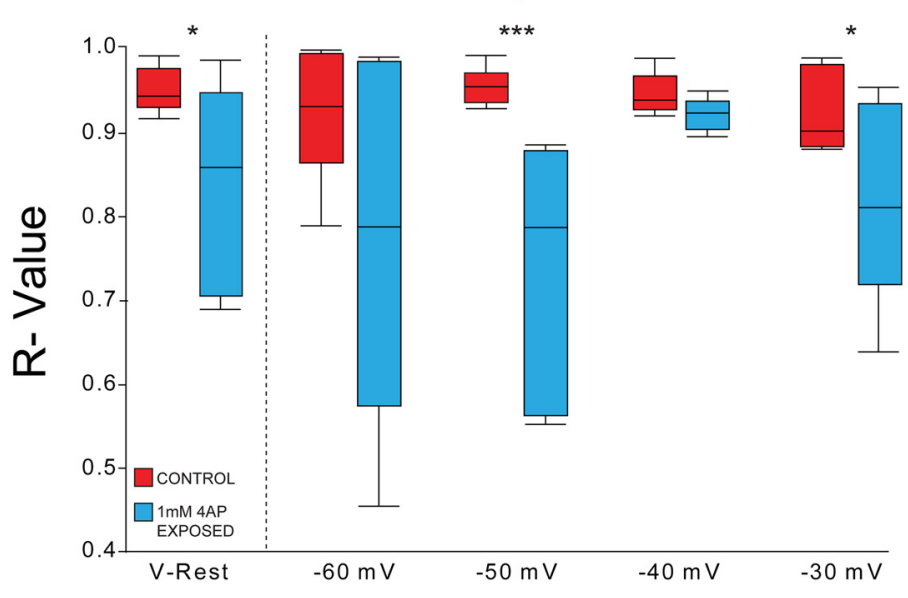

Figure 4. Effects of 4-AP on conserved output. $A$, Overlaid representative traces of $L C$ responses to Stimulus Protocol 1 before and after 4-AP exposure. $R$ values from cross-correlation analyses as in Figure $3 . \boldsymbol{B}$, Box plots show distributions of the $R$ values from whiskers above and below represent the maximum and minimum values. All comparisons made between LCs of the same individual ( $N=7$ and $N=6$ for Stimulus Protocols 1 and 2, respectively), as described in Figure 3. Differences in median $R$ value were tested with Mann-Whitney U tests, and are denoted by asterisks $\left({ }^{* *} p<0.005,{ }^{* *} p<0.01,{ }^{*} p<0.05\right)$.

were not due to changes in $V_{\text {rest }}$ after TEA exposure, current injections were performed from native $V_{\text {rest }}$, as well as standardized baseline voltages ranging from $-60 \mathrm{mV}$ to $-30 \mathrm{mV}$. TEA did not cause a significant change in $V_{\text {rest }}$ (mean $\pm \mathrm{SD}$, control: $-49 \pm 7.03 \mathrm{mV}$, TEA: $-46.3 \pm 10 \mathrm{mV}, p=0.23, N=12$ ). However, TEA did cause significant reduction in mean $R$ value between cells at $V_{\text {rest, }}-50 \mathrm{mV},-40 \mathrm{mV}$, and $-30 \mathrm{mV}$ starting voltages, but not at $-60 \mathrm{mV}$, in both Stimulus Protocols (Fig. $3 A, B)$. At $-60 \mathrm{mV}$, the depolarization in membrane potential due to current injection did not reach the activation voltages of most of the conductances (Ransdell et al., 2012, 2013). Therefore, the $-60 \mathrm{mV}$ data actually demonstrate what happens when these cells are simply passive followers of current injection, and sup- 
port the result that at voltages where conductances are activated, there is a true loss of tuning when a subset of currents are blocked.

To ensure these results are not due to changes in input resistance $\left(R_{\text {in }}\right)$ of cells in TEA, we analyzed relationships between $R_{\text {in }}$ and activity. TEA did not significantly or predictably alter $R_{\text {in }}$ (mean \pm SD, control: $5.03 \pm 2.4 \mathrm{M} \Omega$, TEA: $5.25 \pm 3.28 \mathrm{M} \Omega, p=$ $0.795, N=12$ ). However, we predicted that if loss of conserved activity across LCs with blockade were a result of variable impact of the TEA on $R_{\mathrm{in}}$, then the largest changes in activity between cells should occur with the largest difference in $R_{\text {in }}$ between those two cells. We found no such relationship between change in $R_{\text {in }}$ of individual cells, or the difference in $R_{\text {in }}$ between pairs of cells, with the change in $R$ value in the corresponding cross-correlation (Fig. 3C-F).

Finally, if this is a general phenomenon and not just specific to $I_{\mathrm{HTK}}$, then blocking another conductance in this system also should result in loss of conserved output. We blocked a second $\mathrm{K}^{+}$current, $I_{\mathrm{A}}$, using 4-aminopyridine (4-AP). 4-AP caused the same loss of conserved output in LCs (Fig. 4A) with both Stimulus Protocols, as well as an overall increase in variability of $R$ values (Fig. $4 B$ ).

\section{Discussion}

Evidence for compensation of intrinsic excitability that stabilizes neuronal output has accumulated from nervous systems across phyla, ranging from the cardiac neurons used in this study (Ransdell et al., 2012) to mammalian systems (Desai et al., 1999; Nerbonne et al., 2008). This homeostatic plasticity of intrinsic excitability is likely a critical mechanism underlying neural network dynamics (Davis, 2006; Turrigiano, 2011), and may be involved in disorders of neuronal excitability, such as epilepsy (Echegoyen et al., 2007; Howard et al., 2007). These compensatory responses, and the changes and variability in ionic conductances they induce, are usually the result of pathology, injury, or experimental manipulations that substantially and drastically alter the parameter landscape of these neurons. Yet few of these model systems capture native variability of neurons within unmanipulated networks. So the question remains, do neurons in native networks, that are genetically identical (i.e., cells within the same network), achieve similar output at least in part by differentially tuning the underlying intrinsic conductances via distinct mechanisms? It is this question, independent of compensation, that our study addresses.

We addressed several key components of this question in this study. First, we demonstrated that motor neurons of the cardiac ganglion have similar membrane potential waveforms within the same network, both during intact network activity (Fig. 1B) and as isolated neurons responding to biologically relevant current injection protocols (Fig. 2). This ability to record from unambiguously identified neurons both in intact networks and under experimentally controlled conditions, both of which are crucial to directly test the hypothesis in this paper, represents a key strength of this model system, and how it can contribute uniquely to this question. Second, we measured native current magnitudes of multiple ionic conductances, to ensure that these cells also showed variability in magnitudes of conductances as reported in other systems (Fig. 1C). Not only did these cells show 2-4-fold variability in their underlying conductances, but even cells within the same network showed substantial variability in current magnitudes (Fig. 1D). Third, we ruled out the possibility that the voltage waveforms of isolated cells were passively following external current injection (Fig. 2C,D). Finally, these preconditions allowed us to directly pose the experimental question: does nat- ural variability in underlying current magnitudes result from differentially tuned conductances converging on similar output via distinct solutions? We tested this with a relatively straightforward experimental design. Namely, if output is the result of finely balanced ratios of ionic conductances, then disrupting one conductance should cause each cell to experience a cell-specific detuning, resulting in loss of conserved output. Our results were entirely consistent with the hypothesis: blocking either $I_{\mathrm{HTK}}$ or $I_{\mathrm{A}}$ independently results in loss of similar output across three LC neurons within the same network (Figs. 2, $3 A, B$ ).

Similar manipulations in pacemaker neurons of the stomatogastric ganglion (STG) have yielded different results (Szücs and Selverston, 2006). In the STG, 4AP used to disrupt A-type current in paired pyloric dilator neurons, also reported to have variable conductance magnitudes (MacLean et al., 2005), showed little impact on synchronized activity between these cells (Szücs and Selverston, 2006). These data, although seemingly contradictory, are intriguing as Szücs and Selverston (2006) left chemical and electrical synaptic connectivity, as well as neuromodulatory inputs to these networks (Marder and Thirumalai, 2002) intact during 4AP application. We carefully selected cardiac LCs as a model where we have control over these higher-order network properties, allowing us to focus solely on intrinsic excitability. Interpreted in the context of the Szücs and Selverston (2006) work, convergent output as a result of differentially tuned conductances likely is only one layer of regulation in these systems ensuring synchronized output (Prinz et al., 2004). Work is ongoing to determine whether and how higher-order processes, such as electrical coupling and neuromodulation, ensure that cellular and network output are maintained in patterns necessary for robust function (Grashow et al., 2009, 2010; Marder, 2012).

The most parsimonious explanation for our results is that cells with variable underlying conductances are indeed individually tuned to produce conserved output. We believe these findings provide a clear and unambiguous demonstration of this phenomenon, helping unify a substantial amount of compelling and consistent evidence in multiple model systems, both biological and theoretical (Marder and Taylor, 2011; Marder, 2011; Turrigiano, 2011). It is striking that differential tuning is not simply found across different individuals, but is seen even among cells with in the same individual. One compelling question that arises from these findings is the nature of the developmental and postdevelopmental origin of this variability. Specifically, are individual neurons produced with common underlying conductance relationships that then diverge over time (via compensation), or is variability inherent to the initial development and tuning of a neuron, and maintained across the lifetime of the animal? Answers to these questions will rely on model systems that allow for identified neurons to be characterized effectively over both developmental and postdevelopmental time scales.

\section{References}

Ball JM, Franklin CC, Tobin AE, Schulz DJ, Nair SS (2010) Coregulation of ion channel conductances preserves output in a computational model of a crustacean cardiac motor neuron. J Neurosci 30:8637-8649. CrossRef Medline

Bergquist S, Dickman DK, Davis GW (2010) A hierarchy of cell intrinsic and target-derived homeostatic signaling. Neuron 66:220-234. CrossRef Medline

Cooke IM (2002) Reliable, responsive pacemaking and pattern generation with minimal cell numbers: the crustacean cardiac ganglion. Biol Bull 202:108-136. CrossRef Medline

Davis GW (2006) Homeostatic control of neural activity: from phenomenology to molecular design. Annu Rev Neurosci 29:307-323. CrossRef Medline 
Desai NS, Rutherford LC, Turrigiano GG (1999) Plasticity in the intrinsic excitability of cortical pyramidal neurons. Nat Neurosci 2:515-520. CrossRef Medline

Echegoyen J, Neu A, Graber KD, Soltesz I (2007) Homeostatic plasticity studied using in vivo hippocampal activity-blockade: synaptic scaling, intrinsic plasticity and age-dependence. PLoS One 2:e700. CrossRef Medline

Goaillard JM, Taylor AL, Schulz DJ, Marder E (2009) Functional consequences of animal-to-animal variation in circuit parameters. Nat Neurosci 12:1424-1430. CrossRef Medline

Goldman MS, Golowasch J, Marder E, Abbott LF (2001) Global structure, robustness, and modulation of neuronal models. J Neurosci 21: 5229-5238. Medline

Golowasch J, Goldman MS, Abbott LF, Marder E (2002) Failure of averaging in the construction of a conductance-based neuron model. J Neurophysiol 87:1129-1131. Medline

Grashow R, Brookings T, Marder E (2009) Reliable neuromodulation from circuits with variable underlying structure. Proc Natl Acad Sci U S A 106: 11742-11746. CrossRef Medline

Grashow R, Brookings T, Marder E (2010) Compensation for variable intrinsic neuronal excitability by circuit-synaptic interactions. J Neurosci 30:9145-9156. CrossRef Medline

Hartline DK (1967) Impulse identification and axon mapping of the nine neurons in the cardiac ganglion of the lobster Homarus americanus. J Exp Biol 47:327-340. Medline

Howard AL, Neu A, Morgan RJ, Echegoyen JC, Soltesz I (2007) Opposing modifications in intrinsic currents and synaptic inputs in post-traumatic mossy cells: evidence for single-cell homeostasis in a hyperexcitable network. J Neurophysiol 97:2394-2409. CrossRef Medline

MacLean JN, Zhang Y, Johnson BR, Harris-Warrick RM (2003) Activityindependent homeostasis in rhythmically active neurons. Neuron 37: 109-120. CrossRef Medline

MacLean JN, Zhang Y, Goeritz ML, Casey R, Oliva R, Guckenheimer J, Harris-Warrick RM (2005) Activity-independent coregulation of IA and Ih in rhythmically active neurons. J Neurophysiol 94:3601-3617. CrossRef Medline

Marder E (2011) Variability, compensation, and modulation in neurons and circuits. Proc Natl Acad Sci U S A 108:15542-15548. CrossRef Medline

Marder E (2012) Neuromodulation of neuronal circuits: back to the future. Neuron 76:1-11. CrossRef Medline

Marder E, Taylor AL (2011) Multiple models to capture the variability in biological neurons and networks. Nat Neurosci 14:133-138. CrossRef Medline

Marder E, Thirumalai V (2002) Cellular, synaptic and network effects of neuromodulation. Neural Netw 15:479-493. CrossRef Medline
Nerbonne JM, Gerber BR, Norris A, Burkhalter A (2008) Electrical remodeling maintains firing properties in cortical pyramidal neurons lacking KCND2-encoded A-type K+ currents. J Physiol 586:1565-1579. CrossRef Medline

Prinz AA, Bucher D, Marder E (2004) Similar network activity from disparate circuit parameters. Nat Neurosci 7:1345-1352. CrossRef Medline

Ransdell JL, Nair SS, Schulz DJ (2012) Rapid homeostatic plasticity of intrinsic excitability in a central pattern generator network stabilizes functional neural network output. J Neurosci 32:9649-9658. CrossRef Medline

Ransdell JL, Temporal S, West NL, Leyrer ML, Schulz DJ (2013) Characterization of inward currents and channels underlying burst activity in motor neurons of the crab cardiac ganglion. J Neurophysiol, in press. Medline

Schulz DJ, Goaillard JM, Marder E (2006) Variable channel expression in identified single and electrically coupled neurons in different animals. Nat Neurosci 9:356-362. CrossRef Medline

Schulz DJ, Goaillard JM, Marder EE (2007) Quantitative expression profiling of identified neurons reveals cell-specific constraints on highly variable levels of gene expression. Proc Natl Acad Sci U S A 104:13187-13191. CrossRef Medline

Swensen AM, Bean BP (2005) Robustness of burst firing in dissociated purkinje neurons with acute or long-term reductions in sodium conductance. J Neurosci 25:3509-3520. CrossRef Medline

Szücs A, Selverston AI (2006) Consistent dynamics suggests tight regulation of biophysical parameters in a small network of bursting neurons. J Neurobiol 66:1584-1601. CrossRef Medline

Taylor AL, Goaillard JM, Marder E (2009) How multiple conductances determine electrophysiological properties in a multicompartment model. J Neurosci 29:5573-5586. CrossRef Medline

Tazaki K (1972) The burst activity of different cell regions and intercellular coordination in the cardiac ganglion of the crab, Eriocheir japonicus. J Exp Biol 57:713-726. Medline

Tazaki K, Cooke IM (1983) Separation of neuronal sites of driver potential and impulse generation by ligaturing in the cardiac ganglion of the lobster, Homarus americanus. J Comp Physiol A Neuroethol Sens Neural Behav Physiol 151:329-346. CrossRef

Tazaki K, Cooke IM (1986) Currents under voltage-clamp of burst-forming neurons of the cardiac ganglion of the lobster (Homarus americanus). J Neurophysiol 56:1739-1762. Medline

Turrigiano G (2011) Too many cooks? Intrinsic and synaptic homeostatic mechanisms in cortical circuit refinement. Annu Rev Neurosci 34:89-103. CrossRef Medline

Van Wart A, Matthews G (2006) Impaired firing and cell-specific compensation in neurons lacking nav1.6 sodium channels. J Neurosci 26:71727180. CrossRef Medline 